\title{
Active Scheme to Measure Throughput of Wireless Access Link in Hybrid Wired-Wireless Network
}

\author{
Khondaker M. Salehin and Roberto Rojas-Cessa
}

\begin{abstract}
In this letter, we propose an active scheme to measure the download throughput of an IEEE 802.11 wireless access link in a hybrid wired-wireless network. The proposed scheme is based on sending pairs of probing packets to a wireless end host to determine the smallest and average intra-packet gaps of the probing packets that are used for the estimation of the constant dispersion gap that wireless access creates. We present experimental evaluations of the proposed scheme, and the obtained results show that the proposed scheme achieves high measurement accuracy. Furthermore, we show that the proposed scheme is able to work under the presence of cross traffic along the path.
\end{abstract}

Index Terms-Active measurement, IEEE 802.11, link capacity, available bandwidth, compound probe, wireless throughput.

\section{INTRODUCTION}

The shared-access mechanism of IEEE 802.11 networks along with collisions and channel fading make the measurement of the throughput of a wireless access link complex [1]. Here, throughput is defined as the rate at which data bits can be transmitted in the time taken to transmit a given packet. The throughput is equivalent to the available bandwidth if the maximum packet size that can be transmitted is used. The transmission speed of a packet pair, or a compound probe consisting of a large heading packet $\left(P_{h}\right)$ and a small trailing packet $\left(P_{t}\right)$, over a wireless access link depends on the link capacity, cross-traffic load, the number of retransmission attempts required to access the channel, the time for receiving acknowledgment (ACK), and the delays contributed by the distributed coordination function interframe space (DIFS) and short interframe space (SIFS) [2], as shown in Figure 1.

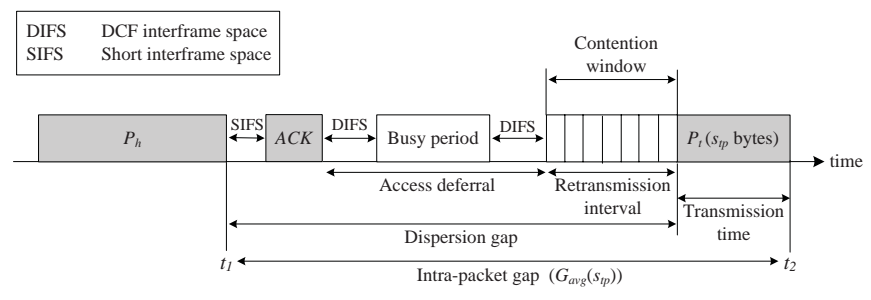

Fig. 1. Intra-packet gap between the heading packet $\left(P_{h}\right)$ and the trailing packet $\left(P_{t}\right)$ over an IEEE 802.11 wireless access link.

For a $P_{t}$ size of $s_{t p}$ bytes, the throughput of the wireless access link is: $T=\frac{s_{t p}}{\left(t_{2}-t_{1}\right)}$, where $t_{1}$ and $t_{2}$ are the arrival times of the last bits of $P_{h}$ and $P_{t}$, respectively, at the wireless

The authors are with Networking Research Laboratory, Department of Electrical and Computer Engineering, New Jersey Institute of Technology, Newark, NJ 07102-1982, USA. Email: \{kms29, rojas\}@ njit.edu. destination host. Therefore, the intra-packet gap between $P_{h}$ and $P_{t}$ is $t_{2}-t_{1}$. However, the intra-packet gap might be affected by cross traffic and heterogeneous link capacities of the wired segment of a hybrid wired-wireless path (Figure 2).

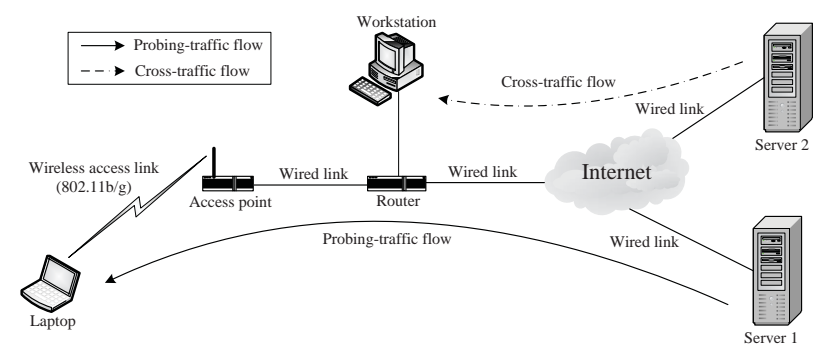

Fig. 2. A hybrid wired-wireless path where a source host (Server 1) is connected to a wireless destination host (Laptop) through multiple wired links and a wireless access link.

Existing schemes based on intra-packet gap [3], [4] and end-to-end delay [1], [5] require that the wireless access link constitute the bottleneck link (i.e., the link with the smallest available bandwidth) of a hybrid wired-wireless path to measure the throughput of the wireless link. If this condition is not satisfied, the accuracy of the schemes may decrease because the probing packets may undergo dispersion created by a bottleneck link located on the wired segment before reaching the wireless access point (AP). Therefore, a scheme to measure the download throughput of a wireless access link that is immune to the bottleneck link location is needed.

In this letter, we propose a scheme to measure the throughput of a wireless access link in a hybrid wired-wireless network where the wireless link is not required to be the bottleneck link of a path under measurement. The scheme uses two compound probes (see Figure 3(a)), with two different $P_{t}$ sizes, $s_{t}=\left\{s_{t a}, s_{t b}\right\}$, to determine the smallest and average intra-packet gaps. The capacity of the wireless access link is then used to calculate the deviations on the expected intra-packet gaps. The deviation indicates the throughput of the wireless access link. Furthermore, the scheme is resilient against the presence of cross traffic on the wired links of the path.

\section{Proposed Scheme For Throughput MEASUREMENT}

In this section, we present the scheme to measure throughput of wireless access link and analyze the conditions required for the sizing of probing packets of the compound probe over a hybrid wired-wireless path. We also introduce an error filtering 




(a)

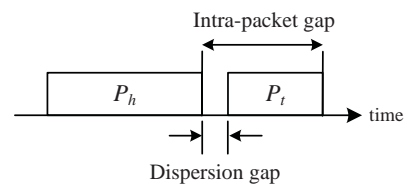

(b)
Fig. 3. A compound probe consisting of a large $P_{h}$ and a small $P_{t}$ a) without and b) with a dispersion gap.

scheme to remove the errors caused by cross traffic in the measurement scheme.

\section{A. Measurement Scheme}

Figure 4 shows the steps of the proposed measurement scheme. Two sets of compound probes are sent from the source host $(s r c)$ to the wireless destination host $(d s t)$ of an end-to-end path using a large $P_{h}$ size, $s_{h}=$ Maximum Transmission Unit (MTU). Upon receiving the compound probes at $d s t$, the scheme determines the smallest intra-packet gap $G_{m i n}\left(s_{t b}\right)$ of the compound probes with $s_{t}=s_{t b}$ bytes, and the smallest and average intra-packet gaps $G_{\min }\left(s_{t a}\right)$ and $G_{a v g}\left(s_{t a}\right)$, respectively, of the compound probes with $s_{t}=s_{t a}$ bytes, where $s_{t a}<s_{t b}$. The reciprocal of the wireless-link capacity $\frac{1}{c_{n}}$ is then determined from the smallest intra-packet gaps of the compound probes. The throughput is calculated as:

$$
T=\frac{s_{t p}}{G_{a v g}\left(s_{t p}\right)}=\frac{s_{t p}}{G_{a v g}\left(s_{t a}\right)-\frac{s_{t a}}{c_{n}}+\frac{s_{t p}}{c_{n}}}
$$

where $s_{t p}$ denotes the packet size for which the throughput is calculated. As stated in (1), the throughput is the ratio between $s_{t p}$ and the intra-packet gap $G_{a v g}\left(s_{t p}\right)$. The gap includes the dispersion gap between $P_{h}$ and $P_{t}$, defined as the gap between the last bit of $P_{h}$ and the first bit of $P_{t}$, as shown in Figure 1. Here, $G_{a v g}\left(s_{t a}\right)-\frac{s_{t a}}{c_{n}}$ is the dispersion gap and $\frac{s_{t p}}{c_{n}}$ is to the transmission time of a $s_{t p}$-byte packet on the wireless link. Further details on (1) can be found in [3].

Because the smallest and average intra-packet gaps of a compound probe might be different on a wireless link, we send multiple compound probes of each $s_{t}$ size in a train for probing the wireless access link.

\section{B. Sizing Probing Packets to Ensure Zero-dispersion Gap}

In a hybrid wired-wireless network with an IEEE 802.11 access link, a compound probe must arrive in the AP with a zero-dispersion gap, as shown in Figure 3(a), so that any dispersion between $P_{h}$ and $P_{t}$ is the product of the access at the wireless link. On the other hand, if a compound probe experiences dispersion, as shown in Figure 3(b), due to cross traffic and heterogeneous link capacities of the wired links [6], the intra-packet gap might not represent the throughput of the wireless link and this adds errors in the measurement.

The sizes of $P_{h}$ and $P_{t}$ to achieve the zero-dispersion gap requirement are determined by the link capacities along the end-to-end path. In a node $i$, if the transmission time of $P_{h}$ on the output link $L_{i+1}$ of node $i$ is smaller than the

\section{Scheme for Throughput Measurement}

\section{1: Set $s_{h}=$ Path MTU}

2 : Set $s_{t}=s_{t b}$, where $s_{t b}$ is determined from (5) or (6)

3 : Send compound probes with $s_{h}$ and $s_{t}$

4 : Get the smallest intra-packet gap $G_{\min }\left(s_{t b}\right)$

5 : Set $s_{t}=s_{t a}$, where $s_{t a}<s_{t b}$

6 : Send compound probes with $s_{h}$ and $s_{t}$

7 : Get the smallest intra-packet gap $G_{\min }\left(s_{t a}\right)$

8: Get the average intra-packet gap $G_{a v g}\left(s_{t a}\right)$

9 : Calculate the capacity $c_{n}, \frac{1}{c_{n}}=\frac{G_{\min }\left(s_{t b}\right)-G_{\min }\left(s_{t a}\right)}{\left(s_{t b}-s_{t a}\right)}$

10 : Calculate the throughput $T$ using (1)

Fig. 4. Proposed scheme to measure the download throughput of a wireless access link in hybrid wired-wireless network.

transmission time of $P_{t}$ on the input link $L_{i}$, the compound probe experiences dispersion. Therefore, to avoid dispersion at node $i$, the packet-size ratio between $P_{h}$ and $P_{t}$ must be equal to or larger than the node's link-capacity ratio [7], or:

$$
\frac{s_{h}}{s_{t}} \geq \frac{c_{i+1}}{c_{i}}
$$

In Figure 5, consider that the link capacities of the endto-end path between src and $d s t$, consisting of multiple wired links, $L_{1}, L_{2}, \ldots, L_{n-1}$, and a wireless link, $L_{n}$, are $c_{1}, c_{2}, \ldots, c_{n}$. Based on (2), the possible dispersion gap at node $i$, where $1 \leq i \leq n-1$, is:

$$
\delta_{i}=\left(\frac{s_{h}}{c_{i+1}}+\Delta-\frac{s_{t}}{c_{i}}\right)+\delta_{i-1}
$$

where $\Delta$ is the additional time required to receive the ACK after the transmission of $P_{h}$ on the wireless link that is considered only when $c_{i+1}$ is a wireless link, and $\delta_{i-1}$ is the dispersion gap at node $i-1$.



Fig. 5. A multiple-hop path with wired (solid line) and wireless (dashed line) links.

The required condition to obtain a zero-dispersion gap in a compound probe at node $n-1$ (AP), in Figure 5, is:

$$
\left(\frac{s_{h}}{c_{n}}+\Delta-\frac{s_{t}}{c_{n-1}}\right)+\left(\frac{s_{h}}{c_{n-1}}-\frac{s_{t}}{c_{n-2}}\right)+\ldots+\left(\frac{s_{h}}{c_{z+1}}-\frac{s_{t}}{c_{z}}\right)=0
$$

where $c_{z}$ is the capacity of the input link of a node $z$ that has the largest link-capacity ratio and that is located after the narrow link (the smallest link capacity of the path), in the direction from src to $d s t$, which also is the closest link to the wireless end host, node $n$, with the largest link-capacity ratio. For example, if two of the nodes after the narrow link 
closest to the wireless end host of a path have the largest linkcapacity ratio, the node located the closest to the wireless end host is selected. However, (4) applies as long as $L_{n}$ is not the narrow link of the path and the largest size of $P_{t}, s_{t}(\max )$, is determined by:

$$
s_{t}(\max )=s_{h} \frac{\sum_{j=z+1}^{n} \frac{1}{c_{j}}+\Delta}{\sum_{j=z}^{n-1} \frac{1}{c_{j}}}
$$

If $L_{n}$ is the narrow link of the path, $s_{t}(\max )$ is simply:

$$
s_{t}(\max )=\left(\frac{s_{h}}{c_{n}}+\Delta\right) c_{n-1}
$$

\section{Filtering of Erroneous Intra-packet Gaps}

In the proposed scheme, the smallest intra-packet gap of a compound probe is inversely proportional to the transmission rate of the wireless link when there is no contention for link access and, therefore, no dispersion in compound probes due to cross traffic. Because the intra-packet gap of a compound probe can have both compression and decompression over the wireless link, due to the limited clock resolution in the operating system at the destination node and the contention by multiple wireless nodes, respectively, we iteratively perform the following statistical analysis to accurately determine the smallest and average intra-packet gaps on the set $(X)$ of intrapacket gaps:

1. Calculate the mean $\bar{x}(j)$ and the standard deviation $\sigma(j)$ of $X$, where $j$ is the iteration number such that $j \geq 1$.

2. If one of the following conditions is satisfied, stop. Else, go to Step 3.
a. $\sigma(j)=0$, for $j \geq 1$.
b. $\sigma(j)=>\sigma(j-1)$, for $j \geq 2$.

3. Discard all data elements in $X$ greater than $\bar{x}(j)$ and go back to Step 1.

The mean value $\bar{x}(1)$ or $\bar{x}(j-1)$ is the smallest intra-packet gap in $X$ if the algorithm terminates after one or $j$ iterations, when $j>1$, respectively.

On the other hand, the average intra-packet gap of $X$ is identified by determining the average of the most frequent intra-packet gap in the sample set where the data elements are distributed with a bin size of $9 \mu \mathrm{s}$. Here, the adopted $9-\mu \mathrm{s}$ bin size is the smallest unit of retransmission interval following a collision on a wireless link as defined in the IEEE 802.11 standard [2], [8].

\section{EXPERIMENTAL RESULTS}

We evaluated the performance of the proposed scheme in a testbed environment over two end-to-end path scenarios: a) single hop and b) multiple hops, as shown in Figure 6. The wireless links in these two scenarios are tested for IEEE 802.11b (11 Mb/s) and IEEE 802.11g (54 Mb/s) transmission rates. The single-hop path consists of a wired link and a wireless link without cross-traffic load along the path. The multiple-hop path has multiple wired links and a wireless access link with $50 \%$ and $75 \%$ cross-traffic loads on the second $\left(L_{2}=155 \mathrm{Mb} / \mathrm{s}\right)$ and third $\left(L_{3}=10 \mathrm{Mb} / \mathrm{s}\right)$ wired links, respectively. In our testbed, the wireless link constitutes the bottleneck link in the single-hop scenario, while the third wired link $\left(L_{3}=10 \mathrm{Mb} / \mathrm{s}\right)$ is the bottleneck link in the multiple-hop scenario. We implemented the proposed scheme as an application for Linux at the end hosts, src and dst, shown in Figure 6.

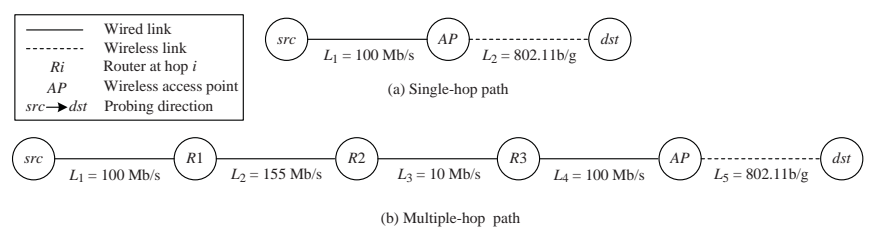

Fig. 6. Hybrid wired-wireless testbed paths: a) single hop and b) multiple hops.

The measurement accuracy of the proposed scheme has been compared against two publicly available tools: a) WBest [4] and b) Iperf [9]. WBest is a state-of-art scheme for measuring throughput of a wireless access link and Iperf is a widely used measurement tool [4]. We performed two sets of measurements for each scheme using an IBM ThinkPad X40 (X40) and a Toshiba Satellite A105 (A105) laptops as dst nodes, which are equipped with Intel Pentium processors, and Intel Pro/Wireless 2200BG and Intel WM3B2200BG network cards, respectively.

We summarize the testbed results in Table I. Here, the values refer to the average of 10 measurements performed by the proposed scheme, WBest, and Iperf. For throughput measurement, the proposed scheme adopted two different set values for $s_{t a}$ and $s_{t b}$, including 8 bytes of UDP header +20 bytes of IP header +14 bytes of MAC header, to be used as $s_{t}$ in the compound probes. Considering the critical packet sizes of the compound probe over the path configurations in Figure 6, with $s_{h}=1500$ bytes and an IEEE $802.11 \mathrm{~g}$ link, we selected $s_{t a}=1392$ bytes and $s_{t b}=1492$ bytes, respectively, for the single-hop scenario, determined by (6), and $s_{t a}=288$ bytes and $s_{t b}=388$ bytes, respectively, for the multiple-hop scenario, determined by (5). Each probing train consisted of 100 compound probes, which we have found to be a suitable number through experimentation, inter-spaced with a constant interval of $100 \mathrm{~ms}$. We used the same number of probing packets in the WBest measurements. Because the probing-train size is not a tunable parameter in Iperf, we ran each measurement iteration for 5 seconds. In WBest and Iperf, 1492-byte packets, including 42 bytes of protocol overhead, were used in the probing train.

In Table I, the Theoretical column shows the theoretical throughputs of a traffic flow with 1450 bytes of User Datagram Protocol (UDP) payload when there is no contention on the IEEE $802.11 \mathrm{~b}$ and $802.11 \mathrm{~g}$ links. These values have been determined in accordance with the IEEE 802.11 standard [10]. The throughput values measured by WBest and Iperf, using 1492-byte probing packets (IP payload size of the probing packets is also 1450 bytes) are shown in the WBest and Iperf columns, respectively. The Proposed scheme column shows the throughput values of IEEE 802.11 links 
TABLE I

MEASUREMENT VALUES

\begin{tabular}{|c|c|c|c|c|c|c|c|c|c|c|c|c|c|}
\hline \multirow[t]{2}{*}{$d s t$} & \multirow{2}{*}{$\begin{array}{l}\text { Path } \\
\text { (hops) }\end{array}$} & \multirow{2}{*}{$\begin{array}{c}\text { Wireless } \\
\text { link } \\
(802.11 x)\end{array}$} & \multicolumn{3}{|c|}{ Intra-packet gaps ( $\mu \mathrm{s})$} & \multirow{2}{*}{$\begin{array}{c}\text { Slope } \\
\frac{1}{c_{n}}\end{array}$} & \multicolumn{4}{|c|}{ Throughput (Mb/s) } & \multicolumn{3}{|c|}{ Error (\%) } \\
\hline & & & $G_{\min }\left(s_{t a}\right), s t d$ & $G_{\min }\left(s_{t b}\right), s t d$ & $G_{a v g}\left(s_{t a}\right)$, std & & Theoretical & WBest & Iperf & Proposed scheme & WBest & Iperf & Proposed scheme \\
\hline X40 & Single & $\overline{\mathrm{b}}$ & $1427,1.2$ & $1516,3.9$ & $1430,0.3$ & 0.81 & 8.50 & 5.98 & 5.96 & 7.67 & 29.65 & 29.88 & 9.76 \\
\hline $\mathrm{X} 40$ & Multiple & b & $551,6.7$ & $628,5.7$ & $556,0.3$ & 0.77 & 8.50 & 4.96 & 5.65 & 7.82 & 41.65 & 33.53 & 8.00 \\
\hline $\mathrm{X} 40$ & Single & $\mathrm{g}$ & $485,2.7$ & $520,1.2$ & $495,8.9$ & 0.35 & 36.02 & 14.27 & 14.25 & 21.88 & 60.38 & 60.44 & 39.26 \\
\hline $\mathrm{X} 40$ & Multiple & $\mathrm{g}$ & $251,9.2$ & $274,1.3$ & $258,0.4$ & 0.23 & 36.02 & 5.24 & 8.19 & 22.28 & 85.45 & 77.26 & 38.15 \\
\hline A105 & Single & $\overline{\mathrm{b}}$ & $1339,56.9$ & $1420,52.7$ & $1419,14.3$ & 0.81 & 8.50 & 5.63 & 5.95 & 7.73 & 33.76 & 30.00 & 9.06 \\
\hline A105 & Multiple & b & $519,7.1$ & $598,6.8$ & $547,14.4$ & 0.79 & 8.50 & 5.02 & 5.59 & 7.74 & 40.94 & 34.24 & 8.94 \\
\hline A105 & Single & $\mathrm{g}$ & $443,30.4$ & $484,13.7$ & $488,45.0$ & 0.41 & 36.02 & 14.84 & 12.8 & 21.92 & 58.80 & 64.46 & 39.14 \\
\hline A105 & Multiple & $\mathrm{g}$ & $222,6.5$ & $243,4.8$ & $242,14.8$ & 0.21 & 36.02 & 5.32 & 8.15 & 23.44 & 85.23 & 77.37 & 34.93 \\
\hline
\end{tabular}

for a packet size $s_{t p}$, with a 1450 -byte IP payload $^{1}$, which is obtained from the measured intra-packet gap values in the Intra-packet gaps column, wireless-link capacity values in the Slope column, and (1). The Intra-packet gaps column contains both the mean and the standard deviation of the measured intra-packet gaps, respectively. The last three columns of Table I show the errors of WBest, Iperf, and the proposed scheme, respectively, in reference to the values in the Theoretical column. The error is, therefore, defined as $\left(\frac{\text { Theoretical throughput }- \text { Measured throughput }}{\text { Theoretical throughput }}\right) \times 100 \%$, where Measured throughput is the throughput of the wireless link measured by WBest, Iperf, and the proposed scheme.

As Table I shows, the error in the throughput values of the proposed scheme, measured on both laptops and testbed paths are significantly smaller than those of the WBest and Iperf values. The errors of the proposed scheme's measurement are about $10 \%$ and $39 \%$ on IEEE $802.11 \mathrm{~b}$ and $802.11 \mathrm{~g}$ links, respectively, over the single-hop path. In the cases of WBest and Iperf measurements, the errors on IEEE $802.11 \mathrm{~b}$ and $802.11 \mathrm{~g}$ links are about $34 \%$ and $64 \%$, respectively. The lower accuracy of WBest and Iperf measurements over the singlehop path may be the result of determining the throughput using the average intra-packet gap of the probing train, which can be affected by large intra-packet gaps.

While the high accuracy of the proposed scheme remains consistent in each path scenario, both WBest and Iperf are not designed to measure throughput on a multiple-hop path where the wireless link does not constitute the bottleneck link. The degradation of measurement accuracy of these schemes in multiple-hop scenario is more evident on the IEEE $802.11 \mathrm{~g}$ link than on the IEEE 802.11b link. For example, the error in the WBest measurement increases from 59\% to $85 \%$ when throughput is measured on the A105 laptop over the multiplehop path using IEEE 802.11g as the wireless access link. Overall, the testbed results show that the proposed scheme outperforms the existing schemes in both path scenarios, even when the wireless access link is the bottleneck link of the end-to-end path. The accuracy of the proposed scheme also remains constant under heavy cross-traffic conditions.

\section{CONCLUSions}

We proposed a scheme to measure download throughput of wireless access links in a hybrid wired-wireless network consisting of IEEE 802.11 links. The scheme is based on

\footnotetext{
${ }^{1}$ Because throughput is calculated using IP payload, the header fields at the network and lower layers are not considered in (1).
}

sending compound probes with two different trailing-packet sizes. We experimentally tested the scheme on single-hop and multiple-hop paths, with different bottleneck-link locations and under different cross-traffic loads on the wired links. The experimental results show that the proposed scheme achieves $90 \%$ and $61 \%$ accuracy on IEEE $802.11 \mathrm{~b}$ and $802.11 \mathrm{~g}$ links, respectively, and it is tolerant to cross-traffic load on the wired links preceding the wireless access link.

\section{REFERENCES}

[1] K. Lakshminarayanan, V.N. Padmanabhan, and J. Padhye, "Bandwidth Estimation in Broadband Access Networks," in Proc. IMC'04, Taormina, Sicily, Italy, Oct. 2004, pp. 314-321.

[2] IEEE Standard 802.11: Wireless LAN Medium Access Control (MAC) and Physical Layer (PHY) Specifications. [Online]. Available: http://standards.ieee.org/getieee802/download/802.11-2007.pdf.

[3] S. Shah, K. Chen, and K. Nahrstedt, "Available Bandwidth Estimation in IEEE 802.11-based Wireless Networks," in Proc. First ISMA Workshop on Bandwidth Estimation (BEst), San Diego, CA, USA, Dec. 2003, pp. $1-3$.

[4] M. Li, M. Claypool, and R. Kinicki, "WBest: A Bandwidth Estimation Tool for IEEE 802.11 Wireless Networks," in Proc. 33rd Annual IEEE Conference on Local Computer Networks, Montreal, Que., Canada, Oct. 2008, pp. 374-381.

[5] A. Johnsson, M. Bjrkman, and B. Melander, "An Analysis of Active End-to-End Bandwidth Measurements in Wireless Networks," in Proc. 4th IEEE/IFIP Workshop on End-to-End Monitoring Technique and Services, Vancouver, BC, Canada, Jul. 2006, pp. 74-81.

[6] K.M. Salehin and R. Rojas-Cessa, "A Combined Methodology for Measurement of Available Bandwidth and Link Capacity in Wired Packet Networks," IET Communications, vol. 4, no. 2, pp. 240-252, 2010.

[7] K. Lai and M. Baker, "Measuring Link Bandwidths Using a Deterministic Model of Packet Delay," in Proc. of ACM SIGCOMM, Stockholm, Sweden, Aug. 2000, pp. 283-294.

[8] D. Vassis, G. Kormentzas, A. Rouskas, and I. Maglogiannis, "The IEEE 802.11g Standard for High Data Rate WLANs," IEEE Network, vol. 19, no. 3, pp. 21-26, 2005.

[9] Iperf. [Online]. Available: http://iperf.sourceforge.net/.

[10] M. Gast. When is 54 Not Equal to 54? A Look at 802.11a, b, and $\mathrm{g}$ Throughput. [Online]. Available: http://www.oreillynet.com/pub/a/wireless/2003/08/08/wireless_throughput. html?page $=2$. 